\begin{tabular}{c} 
International Journal of Engineering \& Technology, $7(3.19)(2018) 20-26$ \\
International Journal of Engineering \& Technology \\
SPC \\
Website: $\frac{w w w . s c i e n c e p u b c o . c o m / i n d e x . p h p / I J E T}{\text { Research paper }}$ \\
\hline
\end{tabular}

\title{
Utilizing Statistical Models for Proposing Hybrid Noise Approach of Image Encryption
}

\author{
Irtefaa A. Neamah ${ }^{1}$, Hind Rustum Mohammed ${ }^{2}$ \\ ${ }^{I}$ Dep. of Mathematics, Faculty of Computer Science and Mathematics, University of Kufa \\ irtefaa.radhi@uokufa.edu.iq \\ ${ }^{2}$ Dep. of Computer Science, Faculty of Computer Science and Mathematics.University of Kufa \\ hindrustum.shaaban@uokufa.edu.iq
}

\begin{abstract}
:
A proposed strategy for an encryption of image based on image noise by statistical models is represented in this paper. An image encryption algorithm has been designed and simulated for some of the common various types of image noise. The proposed method (Hybrid Noise) consisting of a composition of more than one of probability density functions.

Further, the hybrid approach has been compared with some standard noise types such as Gaussian, salt, and pepper, and speckle noise based on some of the performance scales like Peak Signal to Noise Ratio, variance and standard deviation and other comparison tools. The results show that our hybrid method has more security of image with less Peak Signal to Noise Ratio values, which means high noise level.
\end{abstract}

Keywords: Statistical Model, Image Encryption, Image Noise, Impulse Noise, Salt and Pepper Noise, Gaussian Noise, Peak Signal to Noise Ratio (PSNR).

\section{Introduction}

Digital technologies have taken over digital images have become widespread in their applications for example encryption of digital images evolved from conventional techniques to adaptive and lightweight techniques for applications that can run on systems with moderate to low computational capacity Using statistical model (Pal \& Verma, 2017).

The cryptosystem direction has a wide application in the science of data security. Image encryption is ever growing as a highly role in digital image applications (Pal \& Verma, 2017). A vital direction in image processing is the process of image noise as well as de-noising. Sometimes, the drawbacks can be useful in other way. The important of noise image is a good tool for image decryption. In image processing, the beginners discover a different approach by using noise statistics to create a new collection of information from the original image. In image processing, a famous misconception is to ad noise as probability density function (PDF) like Gaussian, uniform, Rayleigh distribution etc. The molding of noise has some applications. (Gravel, Beaudoin,G., \& De Guise, 2004). One of these application is hide all parameters, information, or details of image such as encryption.

Many techniques of encryption always have been used for data of image as well as data of text. To secure the image for private purposes, most of scientific researchers have tried to find best system of cryptosystem. (Mohammed, Hashim, Hussein, \& Mohammed, 2017).

This paper aims to find high level of security for any type of images that will send between users to hide image information from hacking and spying. In addition, to obtain encryption and decryption algorithms use Hybrid Noise Approach based Utilizing statistical Models with high accuracy.
This literature is composed as follows. In Section 2, survey several Some Types of Noise. In section 3, Proposed approach (Hybrid Noise) to seek better security system. In Section 4, Processing Strategy and Results explain the execution of projected technique is assessed by simulation. In Section 5, Comparison and Performance Proposed approach and the conclusion is made in Section 7.

\section{Some Types of Noise}

Frequently we can expect some types of errors and disturbance, as well as the types of image noises. The noise of image can be caused by external image sending electronically. Such as wireless transmission, satellite, or through cable of networks. These noise will appear on transmitted image in different ways based on method. (McAndrew, 2004).

\subsection{Salt and Pepper Noise}

Also, is called impulse (bipolar) noise, shot noise. Its appearance is randomly scattered black or white pixels over the image (McAndrew, 2004). This type of noise has the probability function in the next form:

$f(x)=\left\{\begin{array}{l}p_{p} \text { for } \quad x=p \\ p_{s} \text { for } x=s \\ 0 \text { otherwise }\end{array}\right.$

Where, $p_{p}$ and $p_{s}$ are the probability of the values of pepper and salt respectively. 


\subsection{Gaussian Noise}

The Gaussian noise pdf can be represented by the model:

$$
f(x)=\frac{1}{\sigma \sqrt{2 \pi}} e^{\frac{-(x-\bar{x})^{2}}{2 \sigma^{2}}}
$$

Where $\mathrm{x}$ represent the data of image, $\bar{x}$ is its mean, $\sigma$ is the standard deviation and $\sigma^{2}$ is the variance.

\subsection{Speckle Noise}

The probability density function of speckle noise can be modeled in many ways. One of these ways is using Rayleigh distribution (Tuncer \& Kenneth, 2007). It can be representing by:

$$
f(x)=\frac{x}{\sigma^{2}} e^{\frac{-x^{2}}{2 \sigma^{2}}}
$$

Where $\mathrm{x}$ represents the data and $\sigma^{2}$ is the variance of the distribution.

\section{Proposed Approach (Hybrid Noise )}

The noise is an important factor affecting image quality, which is just as important as the factor of severity because it is an important factor in showing the details in the picture. So, to increase the security of image we would be efficient to use the noise as a cryptography of image. The smart way to do that is composing to create new noise. That would be impossible even to determine the subject. To increase the noise of image we consider a hybrid approach between the Gaussian noise and speckle noise Suppose that $f(x)$ and $g(x)$ represent Gaussian and Rayleigh respectively, and $h(x)=(f \circ g)(x)$, then:

$$
\begin{aligned}
(f \circ g)(x)= & f\left(\frac{x}{\sigma^{2}}\right) e^{\frac{-x^{2}}{2 \sigma^{2}}} \\
& -\left[\left(\frac{x}{\sigma^{2}} e^{\frac{-x^{2}}{2 \sigma^{2}}}\right)-\mu\right]^{2} \\
=\frac{1}{\sigma \sqrt{2 \pi}} e & \frac{-\left[\sigma^{2}\right.}{}
\end{aligned}
$$

Consider:

$$
z=\left[\left(\frac{X}{\sigma^{2}} e^{\frac{-x^{2}}{2 \sigma^{2}}}\right)-\mu\right]
$$

Then, after substituting equation (5) in equation (4) and simplification will become

$h(x)=\frac{1}{\sigma \sqrt{2 \pi}} e^{-\left(\frac{z}{\sigma \sqrt{2}}\right)^{2}}$

\begin{tabular}{|c|c|c|c|c|c|c|c|}
\hline No. & Type & Color & & ize & Gray & \multicolumn{2}{|c|}{ Size } \\
\hline 1. & jpg & & 540 & 960 & 3 & 387 & 960 \\
\hline 2 & tif & & 2063 & 345 & 3 & 258 & 350 \\
\hline 3 & bmp & & 1772 & 284 & 3 & & \\
\hline 4 & png & & 3845 & 512 & 3 & & 52956 \\
\hline
\end{tabular}

This equation represent the proposed hybrid noise.

\section{Sample of Images}

The image sample are images, four of them are gray and the others are color. These images are in different types such as, ( tif), (jpg), (png) and (bmp). In addition, the sizes are numerous also. All their details are shown in the next table: 


\section{Processing Strategy}

This paper dependents a particular strategy for sample of image processing. That represent the standard methods for adding noise for image with our proposed method (Hybrid noise). To know the steps of the MATLAB code, see the following flowchart:

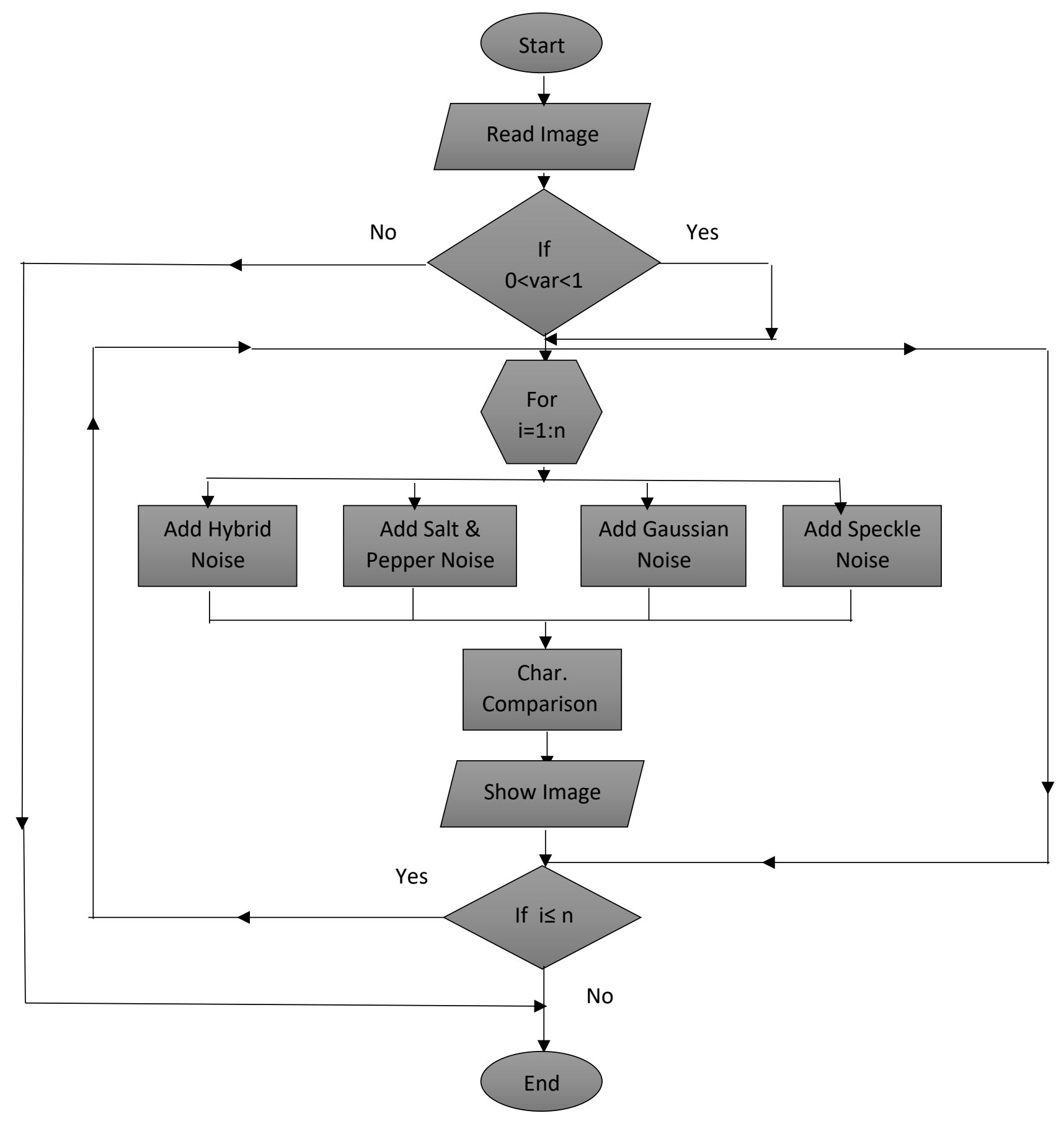

Figure (1): Flowchart of Analyzing Strategy 


\section{Results}

In this section, the suggested method was applied (Hybrid Noise) on gray images and color images, the effect of the method was compared to other noise methods Gaussian, salt and pepper, and speckle noise With the use of Variance Different.The shapes show that the suggested method has the best results in image encryption when focused on probability density functions.

(a): $v=0.2$
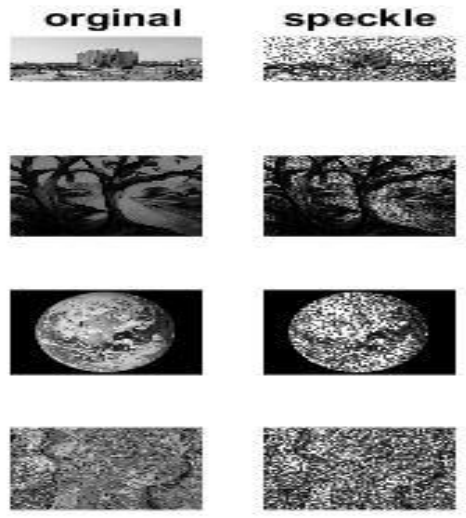
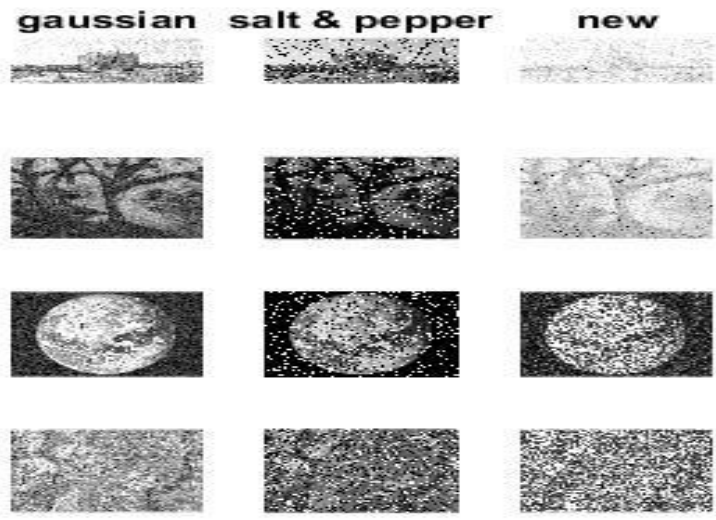

(b) $: v=0.5$
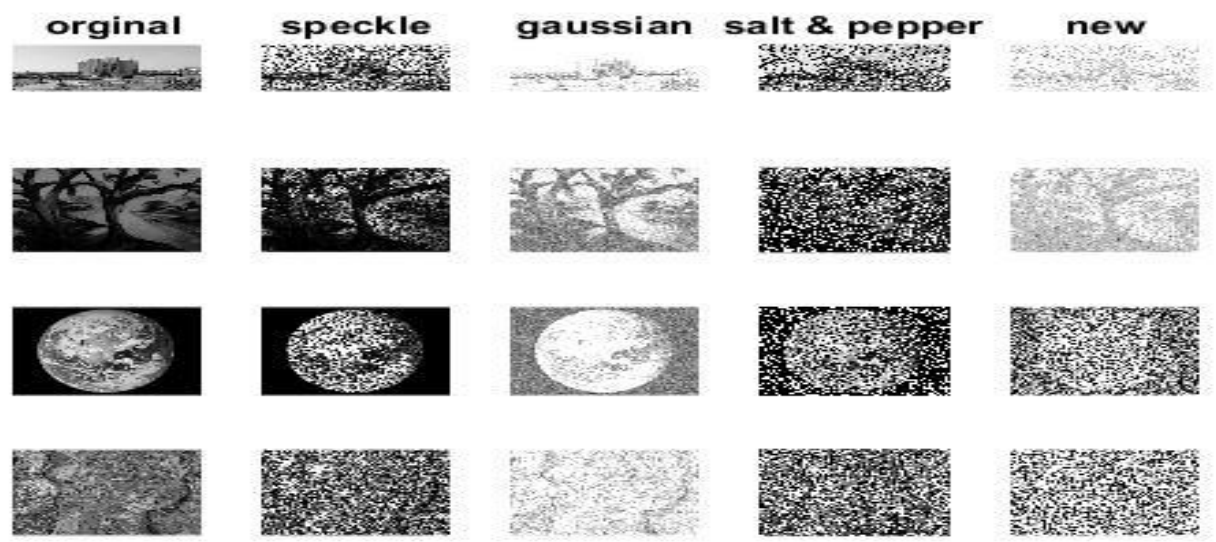

Figure (2): (a) showed Noise Results on Gray Images when variance=0.2. and (b): showed Noise Results with Variances= 0.5 
(c) $: v=0.8$

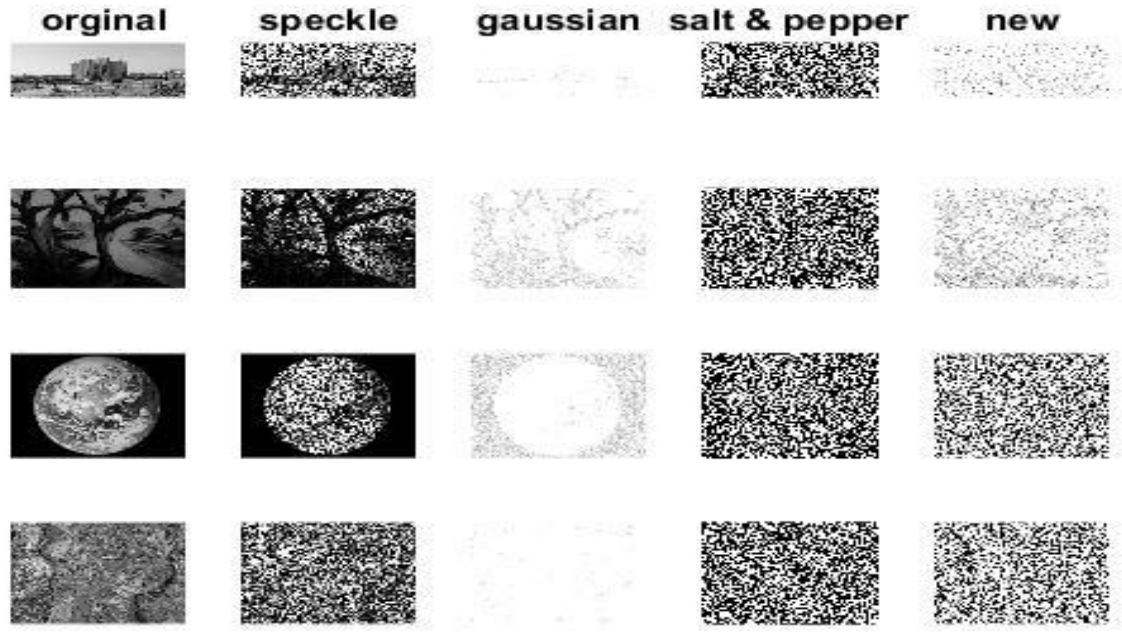

Figure (3): Noise Results on Gray Images when Variance v $=0.8$

(a): $v=0.2$

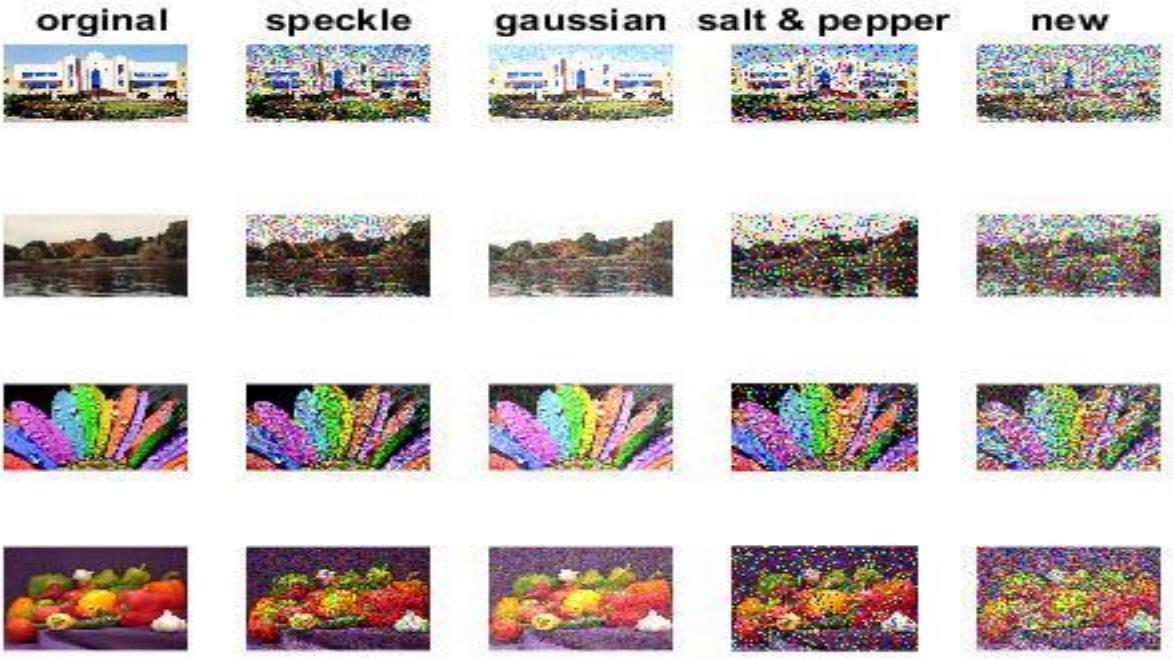

Figure (4): Noise Results on Color Images when Variance v $=0.2$ 
(b): $v=0.5$
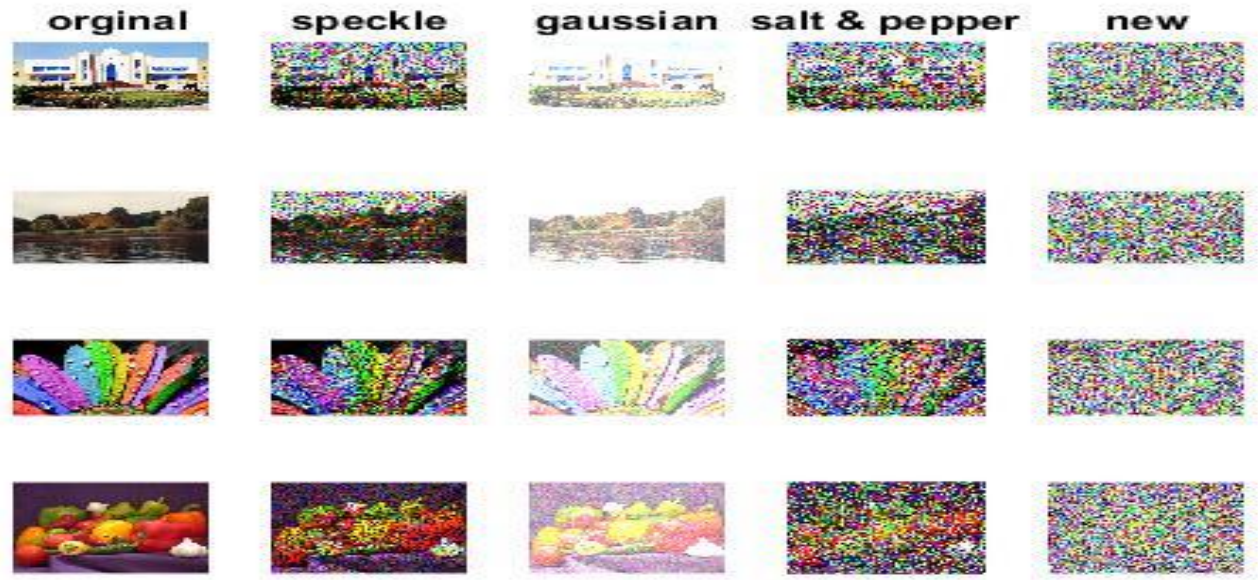

(c) $: v=0.8$
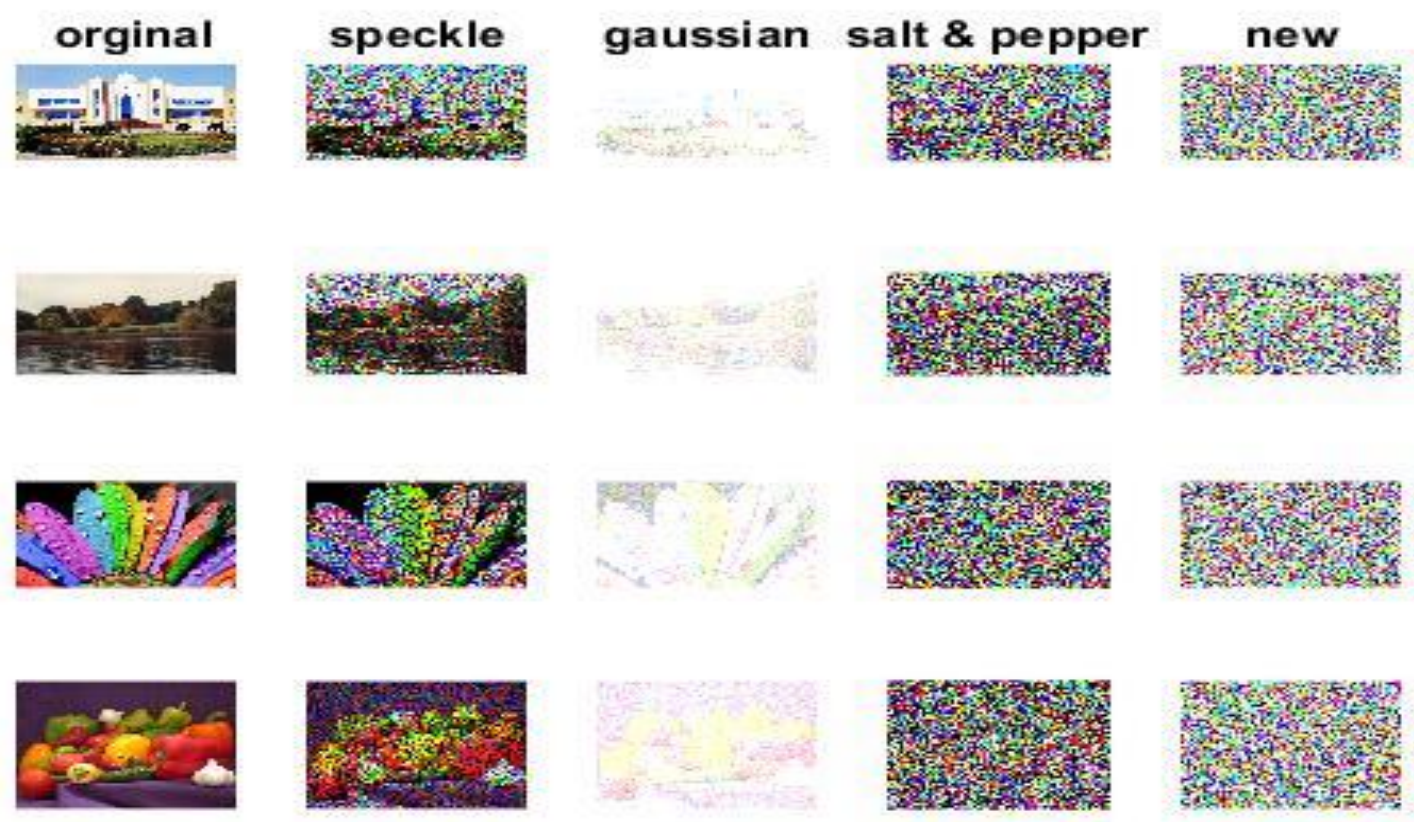

Figure (5): Noise Results on Color Images. (b) Variance v=0.5. (c) Variance v=0.8.

\section{Comparison}

To compare the results for each method, we will take two images only (one is gray and the other is color). The comparison base on some standard scales to know the performance. For example, Peak Signal to Noise Ratio (PSNR). Let $f(i, j)$ and $g(i, j)$ be two images compared and the number of intensity samples is themselves sizes. Suppose $f(i, j)$ is the original image, and $g(i, j)$ is the encrypted image. Then PNSR is defined as follows (Wang \& Bovik, 2009) \& (Wang \& Bovik, Modern Image Quality Assessment, 2006):

When

$$
P N S R=20 \log _{10}\left(\frac{M A X_{f}}{\sqrt{M S E}}\right)
$$

When

$$
M S E=\frac{1}{m n} \sum_{0}^{m-1} \sum_{0}^{n-1}\|f(i, j)-g(i, j)\|^{2}
$$

PSNR is one success scale in image data noisy. To know the quality of image. PNSR is the best tool. When PNSR is smaller that is the information of new image is so much far to the original image. (Albertus, Lukito, Gede, \& Risanuri, 2011) 

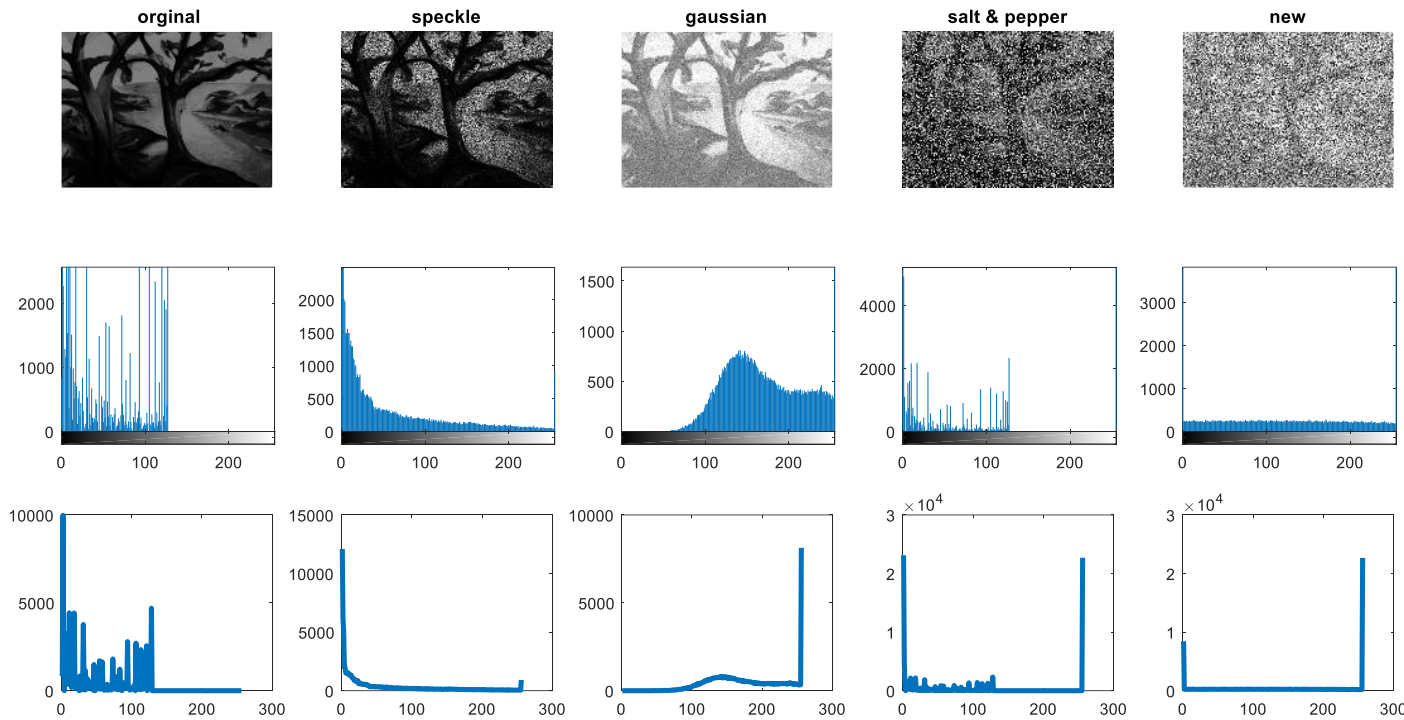

Figure 6: The performance histogram of different methods for one of gray Images when v=0.5

Table 2: showed the final values used for performance evaluation criteria show the main transactions of gray images and color images used in the paper.

\begin{tabular}{cccccc}
\hline Method & Mean & Variance & $\begin{array}{l}\text { Standard } \\
\text { Deviation }\end{array}$ & Minimum & Maximum \\
\hline Origin & 49.0529 & $2.045 * 10^{3}$ & 45.2218 & 0 & 127 \\
Speckle & 50.6119 & $4.9384^{*} 10^{3}$ & 70.2737 & 0 & 255 \\
Gaussian & 162.6888 & $1.0975^{*} 10^{4}$ & 104.7636 & 0 & 13.6501 \\
Salt \& Pepper & 11.6974 & $1.44 * 10^{4}$ & 120.0012 & 0 & 255 \\
Proposed & 232.0550 & 732.3834 & 27.0626 & 112 & 255 \\
\hline
\end{tabular}
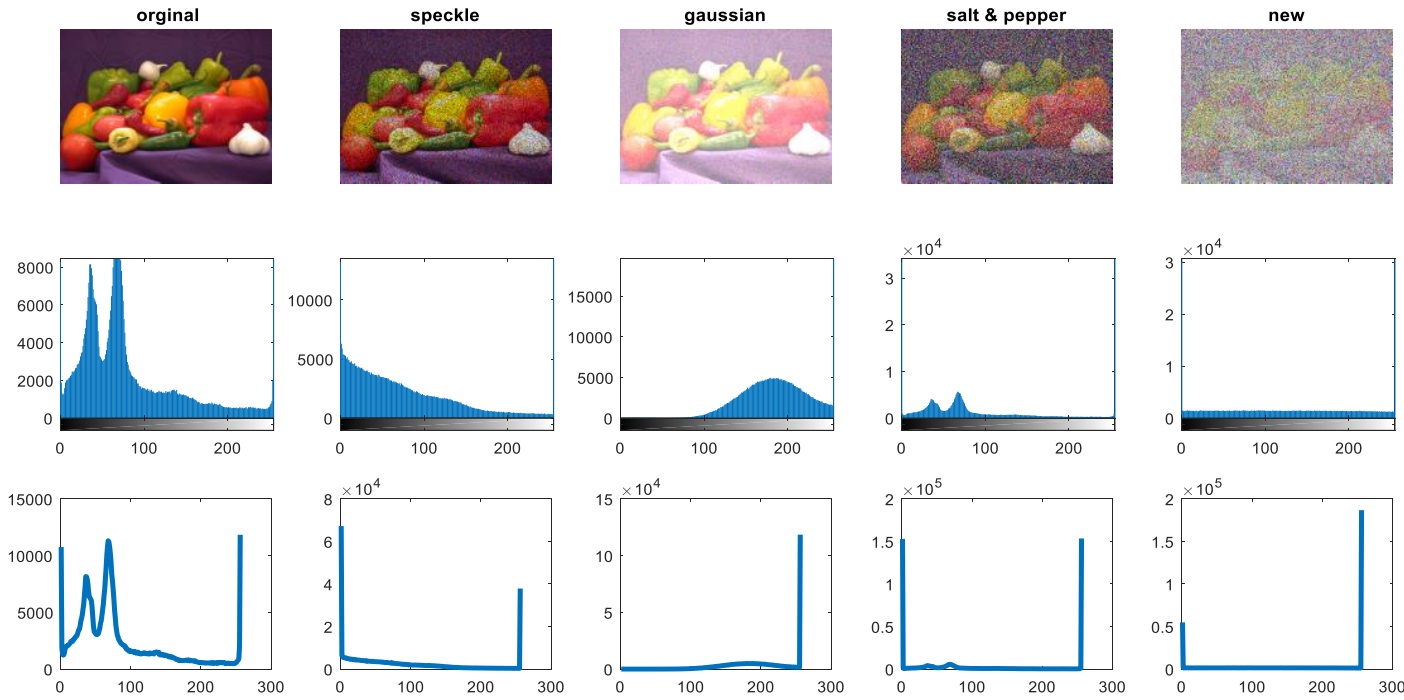

Figure 7: The performance histogram of different methods for one of colored Images when v=0.5

The comparison of the values of the coefficients with the evaluation of the performance of the suggested method is better than the rest of the way and the figures 6 and 7 have shown that. 
Table 3: Performance of clean gray image and the other different methods when $v=0.5$

\begin{tabular}{cccccrr}
\hline Method & Mean & Variance & $\begin{array}{c}\text { Standard } \\
\text { Deviation }\end{array}$ & Minimum & Maximum \\
\hline Origin & 81.6053 & $6.7457^{*} 10^{3}$ & 60.8564 & 0 & 255 & 255 \\
Speckle & 78.4529 & $7.8131^{*} 10^{3}$ & 65.9319 & 0 & 65.9319 \\
Gaussian & 129.9930 & $5.1649^{*} 10^{3}$ & 59.4868 & 0 & 13.3120 & 255 \\
Salt \& Pepper & 90.8151 & $8.747 * 10^{3}$ & 80.8998 & 0 & 255 & 11.6834 \\
Proposed & 119.3709 & $6.4647^{*} 10^{3}$ & 72.7583 & 0 & 10.2727 \\
\hline
\end{tabular}

\section{Conclusion}

In the end of this study, we conclude that the proposed method (hyper noise) has a high level of noise in image. That is mean this noise has ability to encrypt the image, as well as, to high quality to hide all information of image. The new method is developed by composition more than one of statistical model. It is good to encrypt any type of image. The strategy has been run in MATLAB.

a) The proposed method is represent as new method to add noise to image and then encrypting image, for gray and colored image.

b) High quality of this approach based of the quality of common types of image noises and common statistical distributions.

c) From Table 2 the PNSR is (2.7434) for encrypted gray image by hybrid noise. That is mean there is a little information to recognize the origin image.

d) Also, from Table 3, the encrypted color image by proposed method has less PNSR and equal to (10.2727).

e) From the comparison section, we note that the proposed method give high deviation and variance in image data.

\section{References}

[1] Albertus, J. S., Lukito, E. N., Gede, B. S., \& Risanuri, H. (2011) Compression Ratio and Peak Signal to Noise Ratio in Grayscale Image Compression using Wavelet. International Journal of Computer Sci ence and Technology.

[2] Gravel, P., Beaudoin,G., \& De Guise, J. (2004). A Method for Modeling Noise in Medical Images. IEEE TRANSACTIONS ON MEDICAL IMAGING, 1221-1232.

[3] McAndrew, A. (2004). An Introduction to Digital Image Processing with Matlab. Victoria University of Technology, USA.

[4] Mohammed, A. K., Hashim, H., Hussein, A., \& Mohammed, H. (2017). An Algorithm Based on GSVD for Image Encryption. mathematical and Computational Applications.

[5] Pal, G., \& Verma, V. K. (2017). Image Encryption using Adaptive Pixel Masking under Various Noise Attacks. International Journal of Computer Applications, 0975 - 8887.

[6] Tuncer, A. C., \& Kenneth, B. E. (2007). Rayleigh MaximumLikelihood Filtering for Speckle Reduction of Ultrasound Images. IEEE TRANSACTIONS ON MEDICAL IMAGING, 26(5).

[7] Wang, Z., \& Bovik, A. (2006). Modern Image Quality Assessment. Morgan \& Claypool Publishers: Synthesis Lectures on Image, Video, \&Multimedia Processing.

[8] Wang, Z., \& Bovik, A. (2009). Mean squared error: Love it or leave it? a new look at signal fidelity measures. Signal Processing Magazine, IEEE, vol. 26, no. 1, pp. $98-117$, . 